\title{
A GENERALIZATION OF THE LITTLE THEOREM OF PICARD
}

\author{
NICHOLAS C. PETRIDIS \\ Dedicated to the Memory of C. D. Papakyriakopoulos
}

\begin{abstract}
The method used by the author in cooperation with S. I. Goldberg and T. Ishihara in the study of harmonic mappings of bounded dilatation (J. Differential Geometry 10 (4) (1975)) is employed here to derive a Picard type theorem for quasiconformal mappings into spaces which are not necessarily hyperbolic. More precisely: if $N$ is a nonpositively curved Riemannian manifold of dimension $n$ and scalar curvature bounded away from zero, and if $M$ is a complete locally flat Riemannian manifold of dimension $m \geqslant n$, then every harmonic $K$-quasiconformal mapping $f: M$ $\rightarrow N$ is a constant mapping. If, in addition, $M$ and $N$ are Kaehler manifolds then every conformal mapping, $f: M \rightarrow N$ is of rank at most $n-2$. For $m=n=2$ we obtain the classical "little" theorem of Picard.
\end{abstract}

1. Introduction. The "little" theorem of Picard states that a holomorphic mapping of the complex plane to the complex plane with two lacunary values reduces to a constant function. We know that the complex plane $\mathbf{C}$ with two points deleted carries a Kaehler metric of constant negative curvature [7]. From this point of view, the following theorem extends Picard's theorem to mappings of bounded dilatation [3] (see also [4]).

THEOREM A. Let $M$ be a complete connected locally flat Riemannian manifold, and let $N$ be a Riemannian manifold with negative sectional curvatures bounded away from zero. Then, if $f: M \rightarrow N$ is a harmonic mapping of bounded dilatation, it reduces to a constant mapping.

In particular, this theorem holds for harmonic $K$-quasiconformal mappings.

S. Kobayashi, using the notion of hyperbolic manifolds, obtained the following Picard type theorem [7].

THEOREM B. Let $M^{\prime}$ be a complex manifold on which a complex Lie group acts transitively, and let $M$ be a hyperbolic manifold.Then, every holomorphic mapping $f: M^{\prime} \rightarrow M$ is a constant mapping.

Another extension of Picard's theorem, worded more closely to the classical

Received by the editors June 2, 1975 and, in revised form, March 10, 1976.

AMS (MOS) subject classifications (1970). Primary 32H25, 53A99, 30A60.

Key words and phrases. Harmonic mappings, quasiconformal mappings, scalar curvature, Picard's theorem. 
one, was obtained by Fujimoto [2] and Green [5]:

TheOREM C. Let $\mathbf{C}^{m}$ be the m-dimensional complex Euclidean space and let $\mathbf{P}_{n} \mathbf{C}$ be the $n$-dimensional complex projective space. Then every holomorphic mapping $f: \mathbf{C}^{m} \rightarrow \mathbf{P}_{n} \mathbf{C}$ that omits $2 n+1$ hyperplanes in general position must reduce to a constant mapping.

We know that the $n$-dimensional complex projective space with $2 n+1$ hyperplanes in general position deleted is a hyperbolic manifold [6]. Thus, we see that in Theorems A, B, and C the target space is hyperbolic. It is in this sense that the following theorem gives a new extension of Picard's theorem.

THEOREM 1. Let $M$ be a complete locally flat Riemannian manifold of dimension $m$ and let $N$ be a nonpositively curved Riemannian manifold of dimension $n \leqslant m$. If the scalar curvature of $N$ is bounded away from zero, then every harmonic K-quasiconformal mapping $f: M \rightarrow N$ is a constant mapping.

Clearly, $N$ need not be hyperbolic.

An immediate consequence of this theorem is

COROllary 1. Let $M$ be a complete locally flat Riemannian manifold of dimension $m$, and let $N$ be a nonpositively curved Einstein manifold of dimension $2<n \leqslant m$. If $N$ is not locally flat, then every harmonic $K$-quasiconformal mapping $f: M \rightarrow N$ is a constant mapping.

An example is given which shows that the restriction on the dimensions of $M$ and $N$ cannot be dropped.

2. $K$-quasiconformal harmonic mappings. We begin by reviewing some of the results obtained in [3], where detailed proofs can be found.

Let $V$ and $W$ be two Euclidean vector spaces of dimensions $m$ and $n$, respectively. A linear mapping $F: V \rightarrow W$ of $\operatorname{rank} k=\min (m, n)$ maps a $(k-1)$-dimensional sphere to a $(k-1)$-dimensional ellipsoid with semiaxes of lengths $\gamma_{1} \geqslant \gamma_{2} \geqslant \cdots \geqslant \gamma_{k}>0$, where $\gamma_{i}^{2}=\lambda_{i}, i=1,2, \ldots, k$, are the eigenvalues of ${ }^{t} F F: V \rightarrow V$. The ratio $l=\gamma_{1} / \gamma_{k}$ will be called the total dilatation of $F$.

Let $f: M \rightarrow N$ be a $C^{\infty}$ mapping and let $\left(f_{*}\right)_{x}: T_{x} M \rightarrow T_{f(x)} N$ be the induced mapping of tangent spaces at $x$.

Definition 1. If either $\left(f_{*}\right)_{x}=0$ at each $x \in M$ or if the total dilatation $l(x)$ of $\left(f_{*}\right)_{x}$ is bounded above by a constant $K$ on $M$, then $f$ is said to be a $K$ quasiconformal mapping [4].

Using a moving orthonormal frame $\left\{e_{1}, e_{2}, \ldots, e_{m}\right\}$ in $M$ with coframe $\left\{\omega_{1}, \omega_{2}, \ldots, \omega_{m}\right\}$ and corresponding Levi-Civita connection forms $\omega_{i j}, i, j=1$, $2, \ldots, m$, we have

$$
d s^{2}=\omega_{1}^{2}+\omega_{2}^{2}+\cdots+\omega_{m}^{2}
$$

and the structure equations 


$$
d \omega_{i}=\sum \omega_{j} \wedge \omega_{j i}, \quad d \omega_{i j}=\sum \omega_{i k} \wedge \omega_{k j}+\Omega_{i j},
$$

where $\Omega_{i j}$ are the curvature forms. We write

$$
\Omega_{i j}=-\frac{1}{2} \sum R_{i j k l} \omega_{k} \wedge \omega_{l},
$$

where $R_{i j k l}$ are the components of the curvature tensor. The sectional curvature on the section defined by $\left\{e_{i}, e_{j}\right\}$ is given by $K_{i j}=R_{i j i j}$ and the scalar curvature is defined by $R=\sum_{i, j} K_{i j}=\sum R_{i i}$, where $R_{i j}$ is the Ricci curvature tensor defined by $R_{i j}=\sum R_{i k j k}$. We denote the corresponding elements of $N$ with an asterisk. Thus, the Riemannian metric of $N$ is given by $d s^{* 2}=\sum \omega_{a}^{* 2}$. (Here, and in the sequel, the indices run as follows: $i, j, k, \ldots=1,2, \ldots, m$ and $a, b, c, \ldots=1,2, \ldots, n$.) Under the mapping $f$,

$$
f_{*} e_{i}=\sum_{a} A_{i}^{a} e_{a}^{*}, \quad f^{*} \omega_{a}=\sum_{i} A_{i}^{a} \omega_{i},
$$

where $f_{*}$ and $f^{*}$ are the mappings induced by $f$ on tangent vectors and forms.

The covariant differential of $A_{i}^{a}$ is defined by

$$
D A_{i}^{a}=d A_{i}^{a}+\sum A_{j}^{a} \omega_{j i}+\sum A_{i}^{b} \omega_{b a}^{*} \equiv \sum A_{i k}^{a} \omega_{k} \quad \text { (say), }
$$

where $A_{i k}^{a}=A_{k i}^{a}$.

DeFinITION 2. The mapping $f$ is called harmonic [1] if $\sum_{i} A_{i i}^{a}=0$.

Differentiating (2) and using the structure equations (1) in $M$ and $N$ we obtain

$$
\sum_{j} D A_{i j}^{a} \wedge \omega_{j}=\sum_{j} A_{j}^{a} \Omega_{j i}+\sum_{b} A_{i}^{b} \Omega_{b a}^{*}
$$

where

(4) $D A_{i j}^{a} \equiv d A_{i j}^{a}+\sum A_{k j}^{a} \omega_{k i}+\sum A_{i k}^{a} \omega_{k j}+\sum A_{i j}^{b} \omega_{b a}^{*} \equiv \sum A_{i j k}^{a} \omega_{k}$ (say).

The Laplacian of the $C^{\infty}$ function $\left\|f_{*}\right\|^{2}=\Sigma_{a, i}\left(A_{i}^{a}\right)^{2}$ on $M$ is given by

$$
\Delta\left\|f_{*}\right\|^{2}=\sum \nabla^{2}\left\|f_{*}\right\|\left(e_{k}, e_{k}\right)=2 \sum_{i, j, a}\left(\left(A_{i j}^{a}\right)^{2}+A_{i}^{a} A_{i j j}^{a}\right) .
$$

Using (2), (3), (4) and the structure equations, (5) becomes

$$
\begin{aligned}
\frac{1}{2} \Delta\left\|f_{*}\right\|^{2}= & \sum_{a, i, j}\left(A_{i j}^{a}\right)^{2}+\sum_{a, i, j} R_{i j} A_{i}^{a} A_{j}^{a} \\
& -\sum_{a, b, c, d, i, j} R_{a b c d}^{*} A_{i}^{a} A_{j}^{b} A_{i}^{c} A_{j}^{d}+\sum_{a, i, j} A_{i}^{a} A_{j j i}^{a},
\end{aligned}
$$

where the last term $\sum A_{i}^{a} A_{j j i}^{a}$ vanishes if $f$ is harmonic. If $\left\|f_{*}\right\|^{2}$ attains a maximum at $x \in M$, then from (6), 


$$
\sum_{a, i, j} R_{i j} A_{i}^{a} A_{j}^{a}-\sum_{a, b, c, d, i, j} R_{a b c d}^{*} A_{i}^{a} A_{j}^{b} A_{i}^{c} A_{j}^{d}+\sum_{a, i, j} A_{i}^{a} A_{j j i}^{a} \leqslant 0
$$

at $x$.

Let $B_{\alpha}$ be the open ball in $E^{m}$ of radius $\alpha$ and constant negative curvature $-4 / \alpha^{2}$. Then, the metric

$$
d s^{2}=\left(\alpha^{2} / \alpha^{2}-r^{2}\right)^{2} \sum_{i} d x_{i}^{2}
$$

of $B_{\alpha}, r^{2}=x_{1}^{2}+x_{2}^{2}+\cdots+x_{m}^{2}$, is conformally related to the flat metric $d \tilde{s}^{2}=\sum_{i} d x_{i}^{2}$ of $E^{m}$. Let $f: B_{\alpha} \rightarrow N$ be a $C^{\infty}$ mapping which is harmonic with respect to the metrics $\left(d \tilde{s}^{2}, d s^{* 2}\right)$. Such a mapping is not, in general, harmonic with respect to the metrics $\left(d s^{2}, d s^{* 2}\right)$, but the quantity $\sum A_{i}^{a} A_{j j i}^{a}$ of $(f)$ is never negative (see [3, Theorem 4.3]). If by \|\| and \|\|$_{\alpha}$ we denote the norms with respect to the flat metric of $B_{\alpha}$ and its conformally related metric (8), respectively, we have

$$
\left\|f_{*}\right\|_{\alpha}^{2}=\left(\alpha^{2}-r^{2} / \alpha^{2}\right)^{2}\left\|f_{*}\right\|^{2}
$$

The function $\left\|f_{*}\right\|_{\alpha}^{2}$ attains a maximum on the closure of $B_{\alpha}$, but, as we see from (9), it becomes zero on the boundary. Hence, $\left\|f_{*}\right\|_{\alpha}^{2}$ attains a maximum at $x(\alpha)$ in $B_{\alpha}$ unless it is identically zero. Thus, from (7),

$$
-\sum_{a, b, c, d, i, j} R_{a b c d}^{*} A_{i}^{a} A_{j}^{b} A_{i}^{c} A_{j}^{d} \leqslant-\sum_{a, i, j} R_{i j} A_{i}^{a} A_{j}^{a}
$$

at $x(\alpha)$.

From (8), we have $R_{i k i k}=-4 / \alpha^{2}$. Hence, $R_{i j}=-4(m-1) / \alpha^{2} \cdot \delta_{i j}$. Thus, from (10), the following proposition follows.

Proposition 1. The norm $\left\|f_{*}\right\|_{\alpha}$ attains a maximum, denoted by $\left\|f_{*}\right\|_{(\alpha)}$, at some point $x(\alpha) \in B_{\alpha}$, and at this point

$$
-\sum_{a, b, c, d, i, j} R_{a b c d}^{*} A_{i}^{a} A_{j}^{b} A_{i}^{c} A_{j}^{d} \leqslant \frac{4(m-1)}{\alpha^{2}}\left\|f_{*}\right\|_{(\alpha)}^{2},
$$

where $R_{a b c d}^{*}$ are the components of the curvature tensor of $N$ at $f(x)$.

3. Picard's first theorem. Let $\mathbf{C}$ be the entire complex plane, and $a, b$ two points of $\mathbf{C}$. Then, a holomorphic mapping $f: \mathbf{C} \rightarrow \mathbf{C}-\{a, b\}$ is a constant mapping. This is the classical first theorem of Picard. It is known that $\mathrm{C}-\{a, b\}$ can be given a Kaehler metric with curvature bounded above by -4 . In this sense, Theorem 1 gives an extension of Picard's theorem to more general mappings.

In order to prove this theorem we first establish some lemmas. The proof of the following lemma is elementary.

Lemma 1. Let $V$ and $W$ be Euclidean vector spaces, and let $F: V \rightarrow W$ be $a$ 
linear mapping of rank $\geqslant 2$. If $S$ is a two-dimensional subspace of $F(V)$, there is a pair of orthonormal vectors $\left\{X_{1}, X_{2}\right\}$ in $V$ such that the pair $\left\{Y_{1}=F\left(X_{1}\right), Y_{2}\right.$ $\left.=F\left(X_{2}\right)\right\}$ spans $S$ and $Y_{1} \perp Y_{2}$.

LeMma 2. Let $f: M \rightarrow N$ be a K-quasiconformal mapping, and let $X_{1}, X_{2}$ be any two unit vectors of $T_{x}(M)$. If $A_{1}(x)=f_{*}\left(X_{1}\right) \neq 0$ and $A_{2}(x)=f_{*}\left(X_{2}\right) \neq 0$, then $1 / K \leqslant\left\|A_{1}(x)\right\| /\left\|A_{2}(x)\right\| \leqslant K$ for every $x \in M$. Thus, if $\left\|A_{1}(x)\right\| \rightarrow 0$, then $\left\|A_{2}(x)\right\| \rightarrow 0$.

PROof. If $l(x)$ is the total dilatation of $\left(f_{*}\right)_{x}$, and if $\left\|A_{1}(x)\right\| \neq 0,\left\|A_{2}(x)\right\|$ $\neq 0$, then $1 / l(x) \leqslant\left\|A_{1}(x)\right\| /\left\|A_{2}(x)\right\| \leqslant l(x)$. Since $l(x) \leqslant K$ the lemma follows.

Lemma 3. Let $f: M \rightarrow N$ be a $K$-quasiconformal mapping, let $\left\{e_{1}, e_{2}, \ldots, e_{m}\right\}$ be an orthonormal base of $T_{x}(M)$, and let $A_{i}=f_{*}\left(e_{i}\right), i=1,2, \ldots, m$. If $A_{1} \perp A_{2}$, and if at least one of the $\left\|A_{i}\right\|$ is $\geqslant 1$, then

$$
\left\|A_{1} \wedge A_{2}\right\|^{2} /\left\|f_{*}\right\|^{2} \geqslant 1 /\left(K^{2}+(m-1) K^{4}\right) .
$$

Proof. Since $A_{1} \perp A_{2}$, we have $\left\|A_{1} \wedge A_{2}\right\|=\left\|A_{1}\right\| \cdot\left\|A_{2}\right\|$. Hence,

$$
\frac{\left\|A_{1} \wedge A_{2}\right\|^{2}}{\left\|f_{*}\right\|^{2}}=\frac{\left\|A_{2}\right\|^{2}}{1+\sum_{i=2}^{m}\left\|A_{i}\right\|^{2} /\left\|A_{1}\right\|^{2}} .
$$

If $\left\|A_{j}\right\|^{2} \geqslant 1$, then from Lemma 2 ,

$$
\left\|A_{i}\right\|^{2} /\left\|A_{1}\right\|^{2} \leqslant K^{2}, \quad\left\|A_{2}\right\|^{2} \geqslant\left\|A_{j}\right\|^{2} / K^{2} \geqslant 1 / K^{2} .
$$

Substituting in (13), we obtain (12).

LEMMA 4. Let $N$ be a nonpositively curved Riemannian manifold of dimension $n \geqslant 2$, and let $E^{m}$ be $m$-dimensional Euclidean space, where $m \geqslant n$. If at each point of $N$ there is at least one sectional curvature $\leqslant-A$, where $A$ is a positive constant, then a K-quasiconformal harmonic mapping $f: E^{m} \rightarrow N$ is a constant mapping.

Proof. Consider the mapping $f: B_{\alpha} \rightarrow N$. According to Proposition 1, $\left\|f_{*}\right\|_{\alpha}$ attains a maximum $\left\|f_{*}\right\|_{(\alpha)}$ at a point $x(\alpha) \in B_{\alpha}$. By hypothesis, there is a section $S_{f(x(\alpha))} N$ on which $N$ has sectional curvature $\leqslant-A$, where $A$ is a positive constant. If $\left\|f_{*}\right\|_{(\alpha)} \neq 0$, we have $T_{f(x(\alpha))} N=f_{*} T_{x(\alpha)} B_{\alpha}$, since $f_{*}$ is of maximal rank. From Lemma 1, we can then select an orthonormal frame $\left\{X_{1}, X_{2}, \ldots, X_{m}\right\}$ at $x(\alpha) \in B_{\alpha}$ such that the pair $A_{1}=f_{*} X_{1}, A_{2}=f_{*} X_{2}$ spans $S_{f(x(\alpha))}$ and $A_{1} \perp A_{2}$. The sectional curvature of $N$ along $S_{f(x(\alpha))}$ is given by

$$
H_{1,2}=\sum_{a, b, c, d} R_{a b c d}^{*} A_{1}^{a} A_{2}^{b} A_{1}^{c} A_{2}^{d} /\left\|A_{1} \wedge A_{2}\right\|^{2} .
$$

If $-b_{i j} \leqslant 0$ are the sectional curvatures along the sections spanned by $A_{i}, A_{j}$ 
for $i, j=1,2, \ldots, m$, then $H_{1,2} \leqslant-A<0$, and $-b_{i j} \leqslant 0,(i, j) \neq(1,2)$. Hence,

$$
\begin{aligned}
& \sum_{a, b, c, d, i, j} R_{a b c d}^{*} A_{i}^{a} A_{j}^{b} A_{i}^{c} A_{j}^{d} \\
& \leqslant-2 A\left\|A_{1} \wedge A_{2}\right\|^{2}-2 \sum_{(i, j) \neq(1,2)} b_{i j}\left\|A_{i} \wedge A_{j}\right\|^{2} .
\end{aligned}
$$

Inequality (11) of Proposition 1 together with (14) yields

$$
\frac{2(m-1)}{\alpha^{2}}\left\|f_{*}\right\|_{(\alpha)}^{2} \geqslant A\left\|A_{1} \wedge A_{2}\right\|^{2}+\sum_{(i, j) \neq(1,2)} b_{i j}\left\|A_{i} \wedge A_{j}\right\|^{2},
$$

from which

$$
\left(2(m-1) / \alpha^{2}\right)\left\|f_{*}\right\|_{(\alpha)}^{2} \geqslant A\left\|A_{1} \wedge A_{2}\right\|^{2} \text { at } x(\alpha) .
$$

If $\left\|f_{*}\right\|_{(\alpha)}$ is not bounded as $\alpha \rightarrow \infty$, then at least one of the $A_{i}$ has norm $\geqslant 1$ for large values of $\alpha$. Hence, Lemma 3 and (15) yield

$$
2(m-1) / \alpha^{2} \geqslant A /\left(K^{2}+(m-1) K^{4}\right) .
$$

If we let $\alpha \rightarrow \infty$, then (16) implies $A=0$, which contradicts the assumption on $A$. If $\left\|f_{*}\right\|_{(\alpha)}$ is bounded for all $\alpha$, then from (15) and Lemma $2,\left\|A_{i}\right\| \rightarrow 0$ for all $i$ at $x(\alpha)$ as $\alpha \rightarrow \infty$. Thus, $\left\|f_{*}\right\|_{\alpha} \rightarrow 0$ at each point of $B_{\alpha}$ as $\alpha \rightarrow \infty$. But, from (9), $\lim _{\alpha \rightarrow \infty}\left\|f_{*}\right\|_{\alpha}^{2}=\left\|f_{*}\right\|^{2}$ at each point $x \in B_{\alpha}$. Hence, $\left\|f_{*}\right\|=0$.

Proof of Theorem 1. Let $N$ be a nonpositively curved Riemannian manifold with scalar curvature $R$ such that $R \leqslant-B$, where $B$ is a positive constant. Then there is at least one sectional curvature $\leqslant-B / n(n-1)$ at each point of $N$. Let $M$ be a complete connected locally flat Riemannian manifold. We know that the universal covering of $M$ is $E^{m}$. Let $\bar{f}=f \circ \pi: E^{m} \rightarrow N$, where $\pi: E^{m} \rightarrow M$, is the covering map. Then $\left\|\bar{f}_{*}(x)\right\|=\left\|f_{*}(\pi(x))\right\|$. Thus, the theorem follows from Lemma 4.

Proof of Corollary 1 . In an $n$-dimensional Einstein manifold, $n>2$, the scalar curvature $R$ is constant. Since $N$ is not flat, $R<0$, and the proof is a consequence of Theorem 1 .

COROllary 2. Let $M$ and $N$ be Kaehlerian manifolds, which as Riemannian manifolds, are as in Theorem 1. Then every conformal mapping $f: M \rightarrow N$ is of rank at most $n-2$.

Remark. We note here that Picard's classical theorem is contained in the statement of Corollary 2. For then $M=\mathbf{C}$, with the flat metric, $N=\mathbf{C}$ $-\{a, b\}$ with a Kaehler metric of negative curvature bounded away from zero and $n-2=0$.

COROllaRY 3. Let $M$ be a complete connected locally flat Kaehler manifold, and let $\mathbf{C}^{n}$ be the usual $n$-dimensional complex space. Then a conformal mapping 
$f: M \rightarrow \mathbf{C}^{n}$ of rank $n$ cannot miss two parallel hyperplanes.

Proof. Let $L_{1}$ and $L_{2}$ be two parallel hyperplanes missed by $f$. By a linear change of coordinates in $\mathbf{C}^{n}$ the hyperplanes $L_{1}$ and $L_{2}$ may be assumed to be $z_{1}=a$ and $z_{1}=b$. Thus, $\mathbf{C}^{n}-L_{1} \cup L_{2}$ is biholomorphic to $\mathbf{C}^{* n}=(\mathbf{C}$ $-\{a, b\}) \times \mathbf{C}_{2} \times \cdots \times \mathbf{C}_{n}$, where $\mathbf{C}_{i}=\mathbf{C}, i=2, \ldots, n$. We know that $\mathbf{C}$ - $\{a, b\}$ can be given a Kaehler metric $d s_{1}^{2}=2 g d z_{1} d \bar{z}_{1}$ with Gaussian curvature bounded above by -4 . Hence, if the metric of $\mathbf{C}^{* n}$ is defined by

$$
d s^{2}=d s_{1}^{2}+\sum_{i=2}^{n} d z_{i} d \bar{z}_{i}
$$

its scalar curvature is negative and bounded away from zero. Consequently, by Theorem 1 , the mapping is constant.

Remarks. 1. Let $N=\mathbf{C}^{*} \times \mathbf{C}$, where $\mathbf{C}^{*}=\mathbf{C}-\{0,1\}$ with a Kaehler metric $d s_{1}^{2}$ with Gaussian curvature bounded above by -4 , and $\mathbf{C}$ is the complex plane with the flat metric $d s_{2}^{2}=\left|d z_{2}\right|^{2}$. The metric of $N$ is defined as the product metric $d s^{2}=d s_{1}^{2} \times d s_{2}^{2}$. Then, the scalar curvature of $N$ is bounded above by -4 . If $\varphi(z)$ is a nonconstant holomorphic function, the mapping $f$ : $\mathbf{C} \rightarrow N$ defined by $z_{1}=$ const, $z_{2}=\varphi(z)$, where $z_{1}$ is a coordinate of $\mathbf{C}^{*}$, is a nonconstant holomorphic mapping. This example shows that the condition $m \geqslant n$ of Theorem 1 cannot be dropped.

2. The author would like to thank the referee for the following example. Let $N=\mathbf{C}$ with the conformal metric $d s^{2}=\left(1+|z|^{2}\right) d z d \bar{z}$, and $M=\mathbf{C}$ with the flat metric. The curvature of $N$ is strictly negative everywhere, but not bounded away from zero. The identity mapping is trivially conformal, which shows that the restriction on the curvature of $N$ in Theorem 1 is essential.

\section{BIBLIOGRAPHY}

1. J. Eells and J. H. Sampson, Harmonic mappings of Riemannian manifolds, Amer. J. Math. 86 (1964), 109-160. MR 29 \#1603.

2. H. Fujimoto, Extensions of the big Picard's theorem, Tôhoku Math. J. (2) 24 (1972), 415-422. MR 48 \# 11578.

3. S. I. Goldberg, T. Ishihara and N. C. Petridis, Mappings of bounded dilatation of Riemannian manifolds, J. Differential Geometry 10 (1975), 619-630.

4. S. I. Goldberg and T. Ishihara, Harmonic quasiconformal mappings of Riemannian manifolds, Bull. Amer. Math. Soc. 80 (1974), 562-566. MR 49 \#6086.

5. M. Green, Holomorphic maps into complex projective space omitting hyperplanes, Trans. Amer. Math. Soc. 169 (1972), 89-103. MR 46 \#7547.

6. P. Kiernan and S. Kobayashi, Holomorphic mappings into projective space with lacunary hyperplanes, Nagoya Math. J. 50 (1973), 199-216. MR 48 \#4353.

7. S. Kobayashi, Hyperbolic manifolds and holomorphic mappings, Dekker, New York, 1970. MR 43 \#3503.

Department of Mathematics, Eastern Illinois University, Charleston, Illinois 61920 